\title{
Characterization and biological roles of oviduct-specific, oestrogen-dependent glycoprotein
}

\author{
William C. Buhi \\ Department of Obstetrics and Gynecology, University of Florida College of Medicine, \\ Gainesville, FL 32610-0294, USA
}

\begin{abstract}
During late follicular growth, oestrus, fertilization and early embryonic development, the oviduct, under specific hormonal control, produces fluid and contributes secretory macromolecules that optimize the microenvironment for gamete maturation and transport, fertilization and early cleavage-stage embryonic development. This review describes the state of knowledge concerning the physiological and biochemical characterization of the major oviduct secreted protein, the oviduct-specific, oestrogendependent glycoprotein. The identification, localization within the oviduct, binding and association with oocytes, embryos and spermatozoa, hormonal control of gene expression, cloning, gene organization, protein sequences and post-translational modifications of oviduct-specific, oestrogen-dependent glycoprotein are discussed. Identification of biological functions for this glycoprotein, its interactions with spermatozoa, oocytes and embryos and its potential as a paracrine regulator of fertilization and development are also discussed.
\end{abstract}

The mammalian oviduct undergoes significant endocrineinduced morphological, biochemical and physiological changes during the oestrous or menstrual cycle. These changes establish an essential microenvironment within the oviduct for final maturation of gametes, capacitation of spermatozoa, transport of gametes and embryos, fertilization and early cleavage-stage embryonic development. These complex functions are dependent on activities of the ciliated and secretory, non-ciliated epithelia that line the deep longitudinal mucosal folds. These functions and cells are controlled by the ovarian steroids, oestrogen and progesterone. During the follicular phase of development, oestrogen is essential for differentiation of the epithelium and development and maturation of secretory cells concomitant with production of macromolecules. The resultant luminal environment (for reviews, see Hunter, 1988; Leese, 1988; Buhi et al., 2000) consists of proteins and macromolecules derived from either serum transudate or secretory epithelium. In the last decade, it has been recognized that the oviduct and its secretions play roles in various reproductive events. Detection and analyses of de novo synthesized and secreted proteins from the oviduct epithelia have demonstrated a temporal and spatial distribution of macromolecules and differences in protein distribution among species. Furthermore, these analyses have permitted identification of the major secretory product, a high molecular weight, oestrogen-dependent, oviduct-specific glycoprotein.

In this review, the family of glycoproteins identified as

Email: buhiwc@obgyn.ufl.edu the oviduct secretory glycoprotein (pOSP; Buhi et al., 1996), oestrus-associated glycoprotein (EAP; Buhi et al., 1990; King and Killian, 1994), oviduct-specific, oestrusassociated glycoprotein (EGP; Hill et al., 1996a), oviduct glycoprotein (sOP92; Gandolfi et al., 1991), oviductin (Robitaille et al., 1988), MUC-9 (Lagow et al., 1999), glycoprotein GP 215 (Kapur and Johnson, 1988) and oviduct-specific glycoprotein (OGP; Sendai et al., 1995; O'Day-Bowman et al., 1996) will be identified collectively as oviduct-specific, oestrogen-dependent glycoproteins (OGPs). OGPs with different molecular masses and carbohydrate contents are synthesized and released exclusively by oviduct secretory epithelium in all mammals examined with two possible exceptions, rats (Arias et al., 1994) and mares (Buhi et al., 1996). Differences in the expression of OGPs in the infundibulum, ampulla and isthmus have also been demonstrated. Expression of OGPs appears to be regulated and is oestrogen-dependent or oestrogen-associated. However, differences in species (Komiya et al., 1996) and effectors (Sun et al., 1997) are apparent. At least eight OGPs have been cloned and a high degree of conservation in both nucleotide and amino acid sequence demonstrated across species. A universal characteristic of OGPs is their association with the zona pellucida and perivitelline space of oocytes and embryos, with the possible exception of the mouse zona pellucida. A variety of functional roles for OGPs have been proposed, but relatively few studies have been performed. Although these studies in vitro indicate a biological effect of OGP on spermatozoa, eggs and embryos, some studies have contradictory findings. Thus, although specific mechanisms and functions underlying 


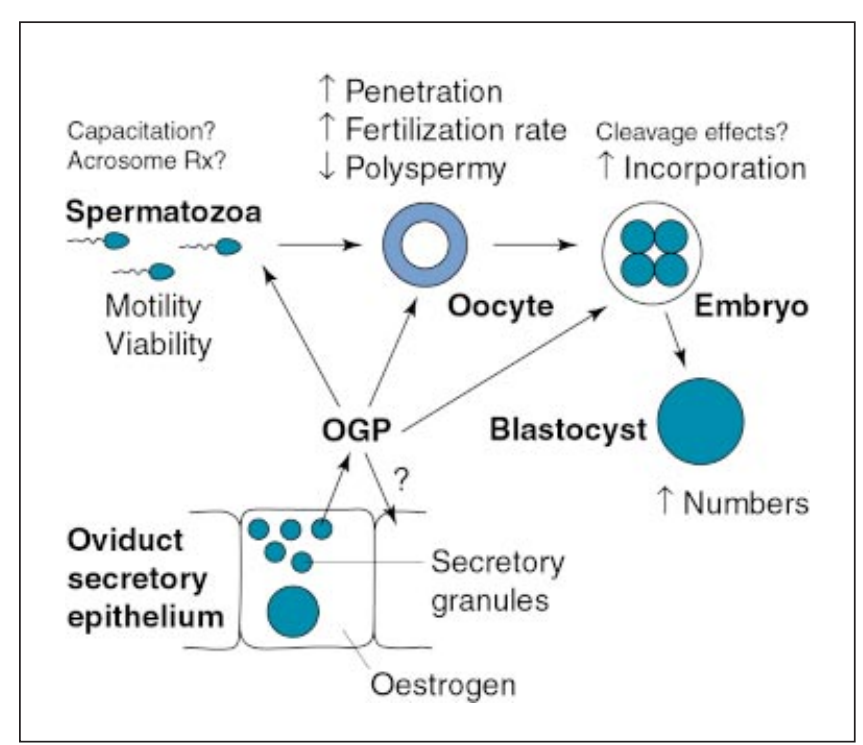

Fig. 1. The oviduct secretory epithelium at oestrus or during the mid-late follicular phase and the influence of oestrogen on synthesis, secretion and function of oviduct-specific, oestrogendependent glycoprotein (OGP) with spermatozoa, oocyte and embryo. Oestrogen transported via the circulation to the epithelium, induces OGP synthesis and secretion. Within the lumen, OGP may interact with spermatozoa to affect motility and viability and has potential to participate in capacitation and the acrosome reaction. Effects of OGP on the ooctye include increased sperm penetration, increased fertilization rates and decreased polyspermy. Potential developmental alterations include changes in cleavage rates and increased number of embryos to the blastocyst stage. Autocrine effects on oviduct epithelium are unknown.

OGP activities remain elusive, the objective of this review is to consider current knowledge of the biochemical and physiological characteristics, localization, hormonal control of expression, cloning, sequence analyses, molecular weight, isoforms, post-translational modifications, and to examine potential functional roles of OGPs with regard to spermatozoa, oocytes and embryos (Fig. 1).

\section{Oviduct-secreted OGPs}

Numerous investigators have proposed that the oviduct expresses specific macromolecular components that interact with gametes or embryos. Pioneering experiments revealed that under oestrogen control, non-ciliated epithelial cells of the rabbit oviduct synthesize a protein de novo that is localized in apical secretory granules. This protein is released into luminal fluid and ultimately becomes part of the egg coat (Oliphant et al., 1984). OGPs have since been identified and characterized using one- and two-dimensional SDS-PAGE, fluorography and immunological detection techniques in other species, including baboons (Verhage et al., 1989), cattle (Boice et al., 1990a), goats (Abe et al., 1995a), hamsters (Robitaille et al., 1988), humans (Verhage et al., 1988), mice (Kapur and Johnson, 1988), pigs (Buhi et al., 1990), rhesus monkeys (Verhage et al., 1997a) and sheep (Sutton et al., 1984). In most species, maximum OGP synthesis and secretion occur during oestrus, the mid- to late follicular phase, fertilization and early cleavage-stage embryonic development (Fig. 2). Furthermore, in pigs, production of OGP is greatest in the ampulla, and second and third greatest in the infundibulum and isthmus, respectively (Fig. 2) (Buhi et al., 1990), and is not detected in any other tissue or fluid compartment, as it is exclusively of oviduct origin. Comparison of proteins revealed OGP to be a high molecular weight glycoprotein with a native undissociated molecular weight of $>1 \times 10^{6}$, whereas the reduced and denatured glycoprotein had molecular masses ranging from 66 to $350 \mathrm{kDa}$. Cow, pig, sheep, human, baboon and hamster OGPs consist of several molecular weight forms and at least two isoforms, acidic and basic molecules. Pig OGP has three major isoforms and two molecular weight variants (Kouba et al., 2000), whereas bovine OGP consists of seven isoforms (Vieira et al., 1999). Immunological crossreactivities, $\mathrm{N}$-terminal amino acid, lectin and carbohydrate analyses demonstrate that, within a species, variants appear to be structurally related. It is unclear why there should be multiple OGP molecular weight variants and isoforms.

\section{Localization of OGPs}

\section{Oviduct}

Immunolocalization and in situ hybridization studies demonstrate that OGPs are products only of non-ciliated oviductal epithelium. Immunoreactive protein is localized in the rough endoplasmic reticula, Golgi and putative secretory granules at apical regions or protrusions primarily in the ampullary epithelium at oestrus, after oestradiol treatment or during the follicular phase of the menstrual cycle. Tissues obtained at the luteal phase, after ovariectomy or after ovariectomy and progesterone treatment demonstrate undetectable or barely detectable OGP (Verhage et al., 1990). Regional differences are apparent as OGPs are not detected immunologically or by in situ hybridization in sheep or mouse isthmus (Kapur and Johnson, 1988; Gandolfi et al., 1991; Murray and DeSouza, 1995). In cattle, sheep, pigs and mice, OGPs have also been immunolocalized to the infundibulum. Why some species do not produce OGP in a specific region is not clear, although lack of production may relate to function within that region.

\section{Oocytes and embryos}

The onset of OGP expression and secretion during late follicular development through early cleavage-stage embryonic development implies an interaction with gametes and embryos. Association or binding of OGP with oocytes from numerous species has been amply demonstrated using immunofluorescent or immunogold localization (for a review, see Buhi et al., 2000). In most species examined, OGPs 
have been localized throughout the zona pellucida, perivitelline space and vitelline or blastomere membrane of oviductal and uterine oocytes and embryos. However, in mice, OGP is found only in the perivitelline space (Kapur and Johnson, 1988).

Distribution of OGPs in the zona pellucida appears uniform and non-dissociable, remaining associated with embryos until day 7 of pregnancy in cattle (Wegner and Killian, 1991) and until pig embryos hatch (Buhi et al., 1993). Although uptake of OGP into baboon (Boice et al., 1990b) and hamster (Kan et al., 1993) blastomeres has been reported, these findings are contradictory to evidence in other species (Gandolfi et al., 1991; Buhi et al., 1993). Taken together, these findings demonstrate that OGP binding and association with ovulated oocytes and embryos is common to all mammalian species examined and indicate that OGPs may play a substantial role as a regulator of fertilization and embryonic development. Because OGPs within species have different molecular weight forms, different isoforms and potentially different post-translational modifications, further studies are necessary to determine whether OGPs associated with zona pellucida, perivitelline space, or vitelline or blastomere membrane consist of different OGP forms, each with a different target and activity. For example, OGP isoforms may differ in their oligosaccharide content, number and distribution of sidechains, and affinity for zona pellucida or vitelline membrane receptors and therefore differ in their ability to regulate biological functions.

\section{Spermatozoa}

The hypothesis that OGPs interact with gametes and embryos is supported in part by the immunolocalization of OGPs with both oocytes and embryos. Immunofluorescent studies with lectin-purified hamster OGP revealed binding to the acrosomal region of spermatozoa before and after capacitation (Boatman and Magnoni, 1995). Furthermore, OGP binding to bovine sperm head and midtail regions differs from that in hamster spermatozoa (King and Killian, 1994). In contrast, studies with human spermatozoa by O'Day-Bowman et al. (1996) did not demonstrate homologous OGP binding. Thus, it remains unclear whether OGP binding to mammalian spermatozoa affects their function.

\section{Hormonal control of expression of OGPs}

In most species examined, expression of OGP mRNA and protein is dependent on the stage of the oestrous cycle, menstrual cycle or early pregnancy and is correlated with circulating concentrations of oestrogen. Regulation of OGP expression appears to show a differential response in species with long oestrous cycles compared with those with short cycles (that is, rodents). In hamsters, differences in expression of OGP mRNA and protein during a normal oestrous cycle were not detected (Komiya et al., 1996). In situ hybridization experiments indicated that OGP mRNA was constitutively expressed, although its signal intensity was attenuated only in ampulla. GnRH analogue treatment decreased oestrogen and OGP mRNA, whereas subsequent administration of oestrogen stimulated the expression of OGP mRNA. Furthermore, oestrogen administered to prepubertal hamsters induced OGP expression (Abe et al., 1998). In contrast to the findings in rodents, as serum oestrogen increases, OGP mRNA in oviduct tissue increases to a maximum value during the late follicular phase in women (Arias et al., 1994) and on days 0-2 of oestrus in pigs (Buhi et al., 1996). Furthermore, during these periods of maximum OGP mRNA expression in oviduct tissue, there are increases in the number of oviduct epithelial apical secretory granules containing OGP and in the amount of OGP protein in oviductal fluid or conditioned culture media. Moreover, during dioestrus or the luteal phase, OGP mRNA and protein are weakly expressed or are undetectable (Fig. 2) (Verhage et al., 1988; Buhi et al., 1991, 1996; Arias et al., 1994).

Ovariectomy and hormone replacement with oestrogen maintains or restores expression of OGP mRNA and protein, whereas addition of progesterone antagonizes the effect of oestrogen, strongly suppressing mRNA and protein. OGP mRNA expression in pig oviduct is significantly greater in the ampulla than in the infundibulum or isthmus throughout the oestrous cycle (Fig. 2b) or after ovariectomy and oestrogen treatment (Buhi et al., 1996). Although the hypothesis that induction and upregulation of OGP expression is oestrogen-dependent is supported by these studies, several laboratories have demonstrated that $\mathrm{LH}$ receptors are present and functional in human, pig and cow oviduct. The presence of $\mathrm{LH}$ receptors and changes in quantity depend on hormonal status and may affect oviduct function (Shemesh, 2001). Sun et al. (1997) suggested that LH has a direct effect on OGP expression as treatment with hCG of bovine epithelium in vitro increases the half-life of OGP mRNA. The functional significance of $\mathrm{LH}$ in other species and in vivo remains to be examined.

\section{Cloning, sequences and modifications of OGPs}

OGP cDNA sequences have been determined in baboons (Jaffe et al., 1996), cows (Sendai et al., 1994), hamsters (Suzuki et al., 1995), humans (Arias et al., 1994), mice (Sendai et al., 1995), pigs (Buhi et al., 1996), rhesus monkeys (Verhage et al., 1997a) and sheep (DeSouza and Murray, 1995a). These OGPs appear to be highly conserved, and share $70-78 \%$ identity and $76-87 \%$ similarity in the overall deduced amino acid sequence. However, the $\mathrm{N}$-terminal region of the mature polypeptide has a high degree of identity $(77-84 \%)$ and similarity $(86-90 \%)$ compared with a low identity $(37-63 \%)$ and similarity (50-75\%) in the C-terminal end. The open reading frames of cDNAs code for a preprotein with a 21 amino acid signal sequence and a total protein of 527-721 amino acids. The predicted molecular masses range from 58.5 to $76.5 \mathrm{kDa}$, 
(a)
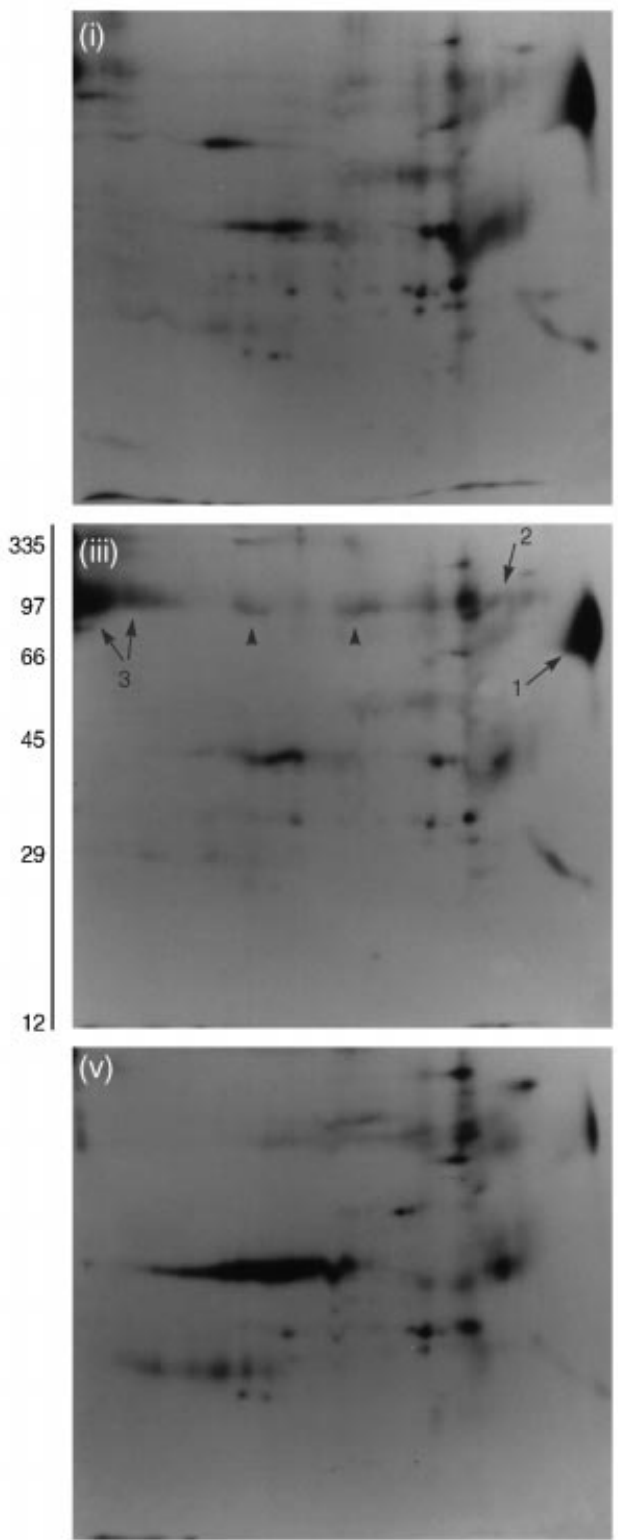

(b)

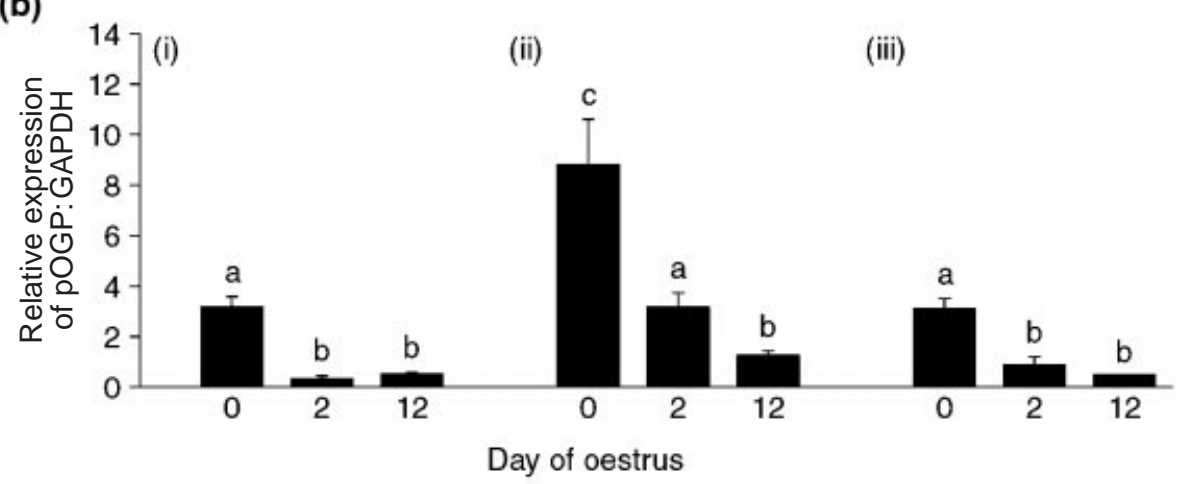

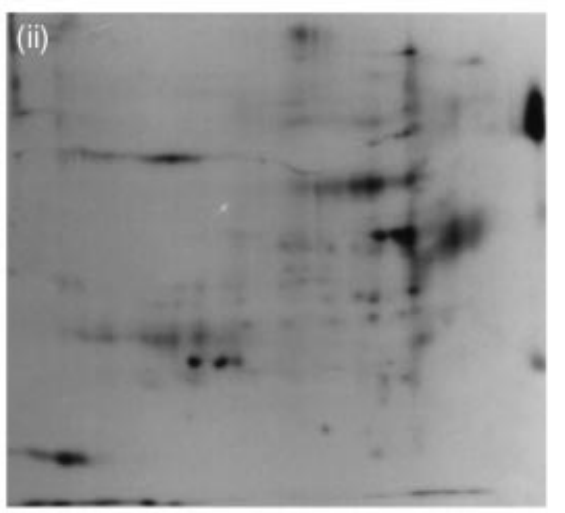
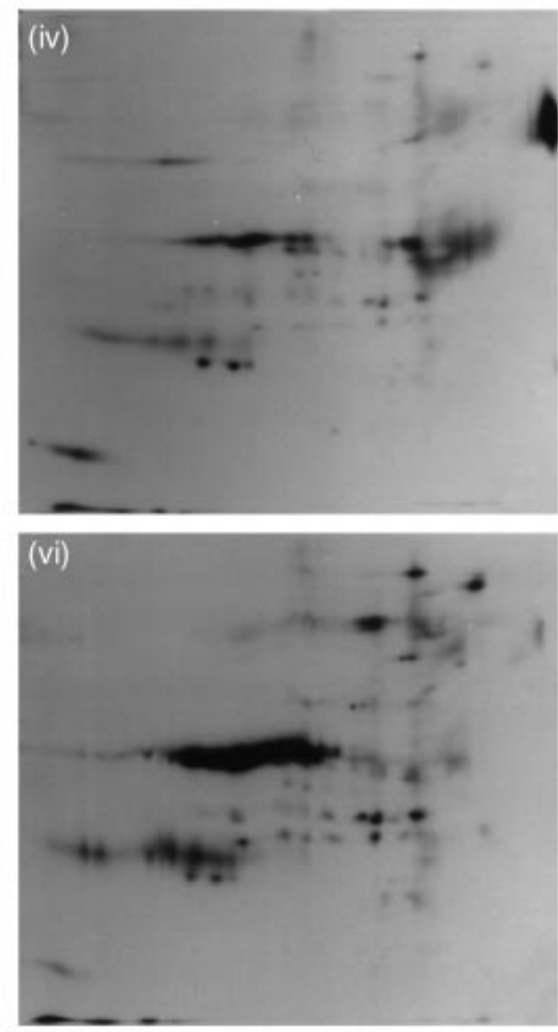

(iii)

Fig. 2. For legend see facing page. 
which is considerably less than the calculated mass of reduced, denatured glycoprotein. Bovine, ovine and pig OGPs are more similar in sequence and length to one another, whereas human, baboon and rhesus monkey OGPs appear more similar to each other.

Major sequence differences are localized in the carboxy terminal region (Buhi et al., 1996), which corresponds to exon 11 of the gene (Verhage et al., 1997b). Significant differences in protein length, amino acids at 141-335, insertions, deletions and truncations are described for this region which contains numerous Ser-Thr residues. The hamster OGP C-terminal region contains a mucin-type, tandem-repeat sequence (six repeats) of 15 amino acids each (Paquette et al., 1995). In contrast, human, baboon and rhesus monkey $\mathrm{C}$-terminal regions contain only four tandem-repeat sequences, whereas those of cattle, sheep and pigs contain incomplete or no tandem-repeat sequences. However, the mouse C-terminal region contains 21 repeat sequences composed of seven amino acids each. Putative $\mathrm{N}$-glycosylation sites, ranging from one to seven, and putative $O$-glycosylation sites, ranging from 10 to 20 (except in mice, in which there are 67 predicted sites), are localized within the carboxy terminal region. In general, tandem repeats are a feature of mucins, although they are exhibited by other proteins. In agreement with the reported characteristics of some OGPs, tandem repeats contain all the $O$-linked glycosylations and are usually found within one exon. Thus, hamster and mouse OGPs may be more closely related to mucins than are other OGPs. Although methods are available for removal of carbohydrate, only one study in vitro (Satoh et al., 1995) investigating the biological role of OGP devoid of carbohydrate has been performed to date. Removal of sialic acid and $N$-linked glycosylation significantly reduces the ability of bovine OGP to maintain sperm viability. Completely deglycosylated OGP is insoluble. Therefore, these glycosylation sites appear to be important for biological activity and for maintaining extracellular solubility. The carbohydrate may also have roles in protection from protease degradation, protein folding and protein-protein interaction. Differences in tandem repeats, the presence and distribution of $N$ - and $O$-linked carbohydrate, the diversity of side chains, and the length of the C-terminal region may confer species specificity, mediate specific recognition events and directly regulate the biological activity of OGPs.

The organization and structure of genomic sequences have been described for human (Jaffe, 1996), mouse (Takahashi et al., 2000) and hamster (Merlen and Bleau, 2000) OGPs. The coding sequence was contained in 11 exons with the signal sequence localized in exons 2 and 3 and the mature peptide found in exons 3-11. A TATAA box was identified in human 5'-flanking sequence, whereas mouse and hamster lacked typical TATA, CAAT and CG boxes. However, atypical nucleotide sequences were identified in both species. An imperfect oestrogen-response element (ERE) was detected in hamsters and mice and ten half-palindromic ERE were located in the putative promoter in mice. Transfection experiments in MCF-7 cells using various sections of the mouse 5 '-flanking region demonstrate that promoter activity for oestrogen responsiveness resides in this region. Additional experiments are required to examine functional analyses, oestrogen receptor binding and other regulatory domains that may control tissue specificity and gene expression.

All OGPs demonstrate a similarity to a growing group of mammalian gene products, which include human cartilage glycoprotein 39 (Hakala et al., 1993) and human macrophage chitotriosidase (Boot et al., 1995), and a group of enzymes (chitinases) not found in mammals. All these proteins belong to family 18 glycosyl hydrolases, proteins of the chitinase gene family. Comparison of the structural organization of genes (Takahashi et al., 2000), protein sequence comparisons and the number and position of conserved cysteine residues (Buhi et al., 1996) in OGPs and mammalian gene products indicates that these proteins have arisen from a common ancestral gene. Hydrophobic cluster analyses and sequence comparisons among all groups indicate that identity and similarity are greater in the $\mathrm{N}$-terminal domain, defined as the catalytic domain, of chitinases. Four residues in chitinases appear to be necessary for activity, but two have been shown by site-directed mutagenesis to be absolute requirements for chitinase activity (Watanabe et al., 1993). Consistent with this finding, all OGPs examined lack an essential glutamic acid residue and chitinase activity. Hydrophobic cluster analysis indicates that amino acids 386-525 in pig OGP correspond to a C-terminal binding domain found in some cellulases and chitinases (Buhi et al., 1996). Owing to divergence in the C-terminal domain, it has been hypothesized that this region constitutes both a binding and species-specific recognition domain.

Post-translational modifications of OGPs may provide

Fig. 2. Temporal and spatial distribution of pig oviduct-specific, oestrogen-dependent glycoprotein (OGP) mRNA and protein in the oviduct. (a) Representative fluorographs of the two-dimensional SDS-PAGE separation of proteins present in culture media conditioned by the infundibulum (i,ii), ampulla (iii,iv) and isthmus ( $v$, vi) from tissues collected on day 0 (i,iii, v) and day 12 (ii,iv, vi). Arrows 1-3 indicate the location of the three major molecular mass and isoelectric forms of pig OGP (previously described as pOSP-E1 (75-85 kDa, pl 4), pOSP-E2 (100 kDa, pl 5-6) and pOSP-E3 (100 kDa, pl 8.5)). Arrowheads identify several minor $100 \mathrm{kDa}$ and isoelectric forms of OGP. Significantly reduced synthesis and secretion of all OGP forms into culture media is shown in the isthmus at day 0 and in all three segments from day 12 tissues. (b) Dot blot hybridization analyses of pig OGP mRNA from the (i) infundibulum, (ii) ampulla and (iii) isthmus on days 0 and 2 of oestrus and day 12 of dioestrus. Blots were probed with a $0.8 \mathrm{~kb}$ fragment of OGP mRNA and quantified by densitometry and relative pOGP:GAPDH expression (mean \pm SD) calculated. Bars with different letters are significantly different ((i) $P<0.01$; (ii,iii) $P<0.05$ ). Location $\times$ day differences in OGP mRNA expression were detected and OGP mRNA abundance in the ampulla was significantly greater than it was in the infundibulum and isthmus, regardless of day (for a review, see Buhi et al., 1996). 
specific biological properties (Satoh et al., 1995) and these modifications may influence protein-protein or protein-cell interactions with sperm membranes, zonae pellucidae, the vitelline membrane of oocytes, or the blastomere membrane of early embryos. Carbohydrate precursor, lectin binding, chemical deglycosylation, enzyme and inhibitor studies indicate that both $\mathrm{N}$ - and $\mathrm{O}$-linked glycosylation occur. Carbohydrates include terminal $\mathrm{N}$-acetylneuraminic acid (sialic acid) in $\alpha(2,3)$ and $\alpha(2,6)$ linkages, galactose, $N$-acetylgalactosamine, $\alpha$-fucose, galactosyl- $\beta(1,3)-N$ acetylgalactosamine, $\mathrm{N}$-acetylglucosamine and mannose (Buhi and Alvarez, 1991; DeSouza and Murray, 1995b; Satoh et al., 1995). Furthermore, metabolic labelling studies have demonstrated that rabbit (Oliphant et al., 1984), pig (Buhi et al., 1990), and hamster (Malette and Bleau, 1993) OGPs are modified by the addition of inorganic sulphate. Although phosphorylation sites are predicted, only pig OGP has been shown to be phosphorylated (Buhi and Alvarez, 1991). Thus, variable isoforms of OGP within a species may be the result of heterogeneous addition of sialic acid residues, other carbohydrates, sulphation or phosphorylation.

\section{Function of OGPs}

Co-incubation of spermatozoa, oocytes or embryos with oviductal fluid, epithelium or conditioned media indicate that OGPs are involved in a variety of roles during fertilization and early embryonic development (Fig. 1). The physiological significance of OGPs binding to oocytes, spermatozoa and embryos is not known, but apparent targets are the zonae pellucidae, and the perivitelline space and membranes of oocytes or blastomeres. The fact of OGP binding to spermatozoa indicates, but does not establish, an effect on sperm activity. Moreover, in species in which OGP has not been shown to bind spermatozoa, effects on sperm activity have been demonstrated. Treatment of bovine spermatozoa with enriched or semi-purified OGP significantly increased capacitation and ability to fertilize bovine oocytes (King et al., 1994) or increased motility and viability when added in a dose-dependent manner (Abe et al., 1995b). Similarly, pig spermatozoa showed increased viability with semi-purified OGP at lower concentrations (McCauley et al., 2001). OGP added in increasing concentrations to pig spermatozoa during a homologous IVF maintained high penetration rates at low concentrations $\left(<20 \mu \mathrm{g} \mathrm{m}^{-1}\right)$ with a significant decrease in polyspermy (Kouba et al., 2000). However, with increased OGP concentrations $\left(\geqslant 50 \mu \mathrm{g} \mathrm{ml}^{-1}\right)$, sperm penetration and binding were significantly reduced.

Co-incubation of the homologous OGP and human spermatozoa and hemizona or hamster spermatozoa and ovarian oocytes demonstrated increased sperm binding and penetration (Boatman and Magnoni, 1995; O'Day-Bowman et al., 1996). Despite high homology between human and baboon OGP, human sperm binding to hemizona was decreased with addition of the heterologous baboon OGP, indicating the importance of a homologous system
(O'Day-Bowman et al., 1996). Incubation of ovine spermatozoa, oocytes and enriched OGP during fertilization showed significant increases in cleavage rate and in numbers of blastocysts (Hill et al., 1996b). However, addition of OGP during culture after IVF showed no effect on cleavage rates (Hill et al., 1996a). Similarly, during pig IVF, no effects on cleavage rates were noted, but increased numbers of embryos at the blastocyst stage were found with OGP treatment at fertilization (Kouba et al., 2000). Embryotrophic effects of OGP in the development of bovine zygotes to blastocysts have not been detected. In contrast, pig OGP added to zygotes fertilized in vivo showed a significant increase in incorporation of radiolabelled amino acid into protein at the four-cell stage (Wallenhaupt et al., 1996). Taken together, these results support a possible role for OGP during embryonic development in vitro and indicate a window of importance during fertilization and first cleavage events.

OGP pretreatment of spermatozoa alone, before fertilization, has produced contradictory results. Bovine spermatozoa preincubated with OGP showed increased capacitation and fertilization rates compared with controls (King et al., 1994). However, a second study in cattle (Martus et al., 1998) found that OGP pretreatment of spermatozoa did not alter fertilization rates. In pig spermatozoa treated with OGP concentrations lower than those in bovine studies, there was a significant increase in the penetration rate compared with that in controls (McCauley et al., 2001) and an increase in polyspermy. Differences in study design, protein purity and concentration, and sperm concentration may explain these disparities.

OGP pretreatment of bovine and porcine ovarian oocytes alone, before fertilization, showed positive effects. In pigs, high penetration and fertilization rates were maintained and polyspermy was significantly reduced when oocytes were treated with low OGP concentrations (McCauley et al., 2001), identical to results after both oocytes and spermatozoa were treated with OGP (Kouba et al., 2000). Similarly, bovine oocytes preincubated with OGP demonstrated significant increases in fertilization rates (Martus et al., 1998). These results indicate an effect of OGP on the oocyte. Furthermore, studies by Martus et al. (1998) and Kouba et al. (2000) extend this concept by demonstrating that biological effects of OGP are blocked with inclusion of specific anti-OGP antibody. Taken together, these studies demonstrate that OGPs have biological effects on both spermatozoa and eggs. However, overall results indicate that the effects of OGPs on reproductive events are mediated primarily through interactions with the oocyte, which may extend into embryonic development.

\section{Conclusion}

OGP has been demonstrated to be the major secretory glycoprotein and to be expressed exclusively by the oviduct. This protein, under oestrogen control during oestrus and the follicular phase of the menstrual cycle, is conserved across 
all species examined. OGP binding to the extracellular matrix, the zona pellucida, and membranes of gametes and embryos indicates a potential biological role during fertilization and development. Functional studies indicate that OGPs affect sperm capacitation, motility and viability. Moreover, evidence indicates that OGPs have direct effects on egg penetration, fertilization and development that may be mediated through interactions with the oocyte.

\section{References}

Key references are identified by asterisks.

Abe H, Onodera M and Sugawara S (1995a) Immunological detection and characterization of an estrus-associated antigen in the goat oviduct Journal of Experimental Zoology 272 134-141

Abe H, Sendai Y, Satoh T and Hoshi H (1995b) Bovine oviduct-specific glycoprotein: potent factor for maintenance of viability and motility of bovine spermatozoa in vitro. Molecular Reproduction and Development 42 226-232

Abe H, Satoh T and Hoshi H (1998) Primary modulation by oestradiol of the production of an oviduct-specific glycoprotein by the epithelial cells in the oviduct of newborn golden hamsters Journal of Reproduction and Fertility 112 157-163

Arias EB, Verhage HG and Jaffe RC (1994) Complementary deoxyribonucleic acid cloning and molecular characterization of an estrogendependent human oviductal glycoprotein Biology of Reproduction $\mathbf{5 1}$ 685-694

Boatman DE and Magnoni GE (1995) Identification of a sperm penetration factor in the oviduct of the golden hamster Biology of Reproduction $\mathbf{5 2}$ 199-207

Boice ML, Geisert RD, Blair RM and Verhage HG (1990a) Identification and characterization of bovine oviductal glycoproteins synthesized at estrus Biology of Reproduction 43 457-465

Boice ML, McCarthy TJ, Mavrogianis PA, Fazleabas AT and Verhage HG (1990b) Localization of oviductal glycoproteins within the zona pellucida and perivitelline space of ovulated ova and early embryos in baboons (Papio anubis) Biology of Reproduction 43 340-346

Boot RG, Renkema GH, Strijland A, van Zonneveld AJ and Aerts JM (1995) Cloning of a cDNA encoding chitotriosidase, a human chitinase produced by macrophages Journal of Biological Chemistry $\mathbf{2 7 0}$ 26 252-26256

Buhi WC and Alvarez IM (1991) Phosphorylation and glycosylation of the 75-85 K Mr estrogen-induced oviductal secretory protein Biology of Reproduction 42 (Supplement 1) 162

Buhi WC, Alvarez IM, Sudhipong V and Dones-Smith MM (1990) Identification and characterization of de novo-synthesized porcine oviductal secretory protein Biology of Reproduction 43 929-938

Buhi WC, Bazer FW, Alvarez IM and Mirando MA (1991) In vitro synthesis of oviductal proteins associated with estrus and $17 \beta$-estradiol-treated ovariectomized ewes Endocrinology 128 3086-3095

Buhi WC, O'Brien B, Alvarez IM, Erdos G and Dubois D (1993) Immunogold localization of porcine oviductal secretory proteins within the zona pellucida, perivitelline space, and plasma membrane of oviductal and uterine oocytes and early embryos Biology of Reproduction 48 1274-1283

Buhi WC, Alvarez IM, Choi I, Cleaver BD and Simmen FA (1996) Molecular cloning and characterization of an estrogen-dependent porcine oviductal secretory glycoprotein Biology of Reproduction $\mathbf{5 5}$ 1305-1314

*Buhi WC, Alvarez IM and Kouba AJ (2000) Secreted proteins of the oviduct Cells Tissues Organs 166 165-179

DeSouza MM and Murray MK (1995a) An estrogen-dependent secretory protein, which shares identity with chitinases, is expressed in a temporally and regionally specific manner in the sheep oviduct at the time of fertilization and embryo development Endocrinology 136 2485-2496

DeSouza MM and Murray MK (1995b) An estrogen-dependent sheep oviductal glycoprotein has glycan linkages typical of sialomucins and does not contain chitinase activity Biology of Reproduction 53 $1517-1526$

Gandolfi F, Modina S, Brevini TAL, Galli C, Moor RM and Lauria A (1991) Oviduct ampullary epithelium contributes a glycoprotein to the zona pellucida, perivitelline space and blastomeres membrane of sheep embryos European Journal of Basic and Applied Histochemistry 35 383-392

Hakala BE, White C and Recklies AD (1993) Human cartilage gp-39, a major secretory product of articular chondrocytes and synovial cells, is a mammalian member of the chitinase protein family Journal of Biological Chemistry $26825803-25810$

Hill JL, Walker SK, Brown GH and Nancarrow CD (1996a) The effects of an ovine oviductal estrus-associated glycoprotein on early embryo development Theriogenology 46 1367-1377

Hill JL, Walker SK, Brown GH and Nancarrow CD (1996b) The effects of an estrus-associated oviductal glycoprotein on the in vitro fertilization and development of ovine oocytes matured in vitro. Theriogenology $\mathbf{4 6}$ 1379-1388

Hunter RHF (1988) The Fallopian Tubes: Their Role in Fertility and Infertility Springer-Verlag, Berlin

Jaffe R (1996) Characterization of the human oviductal glycoprotein gene Biology of Reproduction 54 (Supplement 1) 74

Jaffe RC, Arias EB, O'Day-Bowman MB, Donnelly KM, Mavrogianis PA and Verhage HG (1996) Regional distribution and hormonal control of estrogen-dependent oviduct-specific glycoprotein messenger ribonucleic acid in the baboon (Papio anubis) Biology of Reproduction 55 421-426

Kan FWK, Roux E and Bleau G (1993) Immunolocalization of oviductin in endocytic compartments in the blastomeres of developing embryos in the golden hamster Biology of Reproduction 48 77-88

Kapur RP and Johnson LV (1988) Ultrastructural evidence that specialized regions of the murine oviduct contribute a glycoprotein to the extracellular matrix of mouse oocytes Anatomical Record 221 720-729

King RS and Killian GJ (1994) Purification of bovine estrus-associated protein and localization of binding on sperm Biology of Reproduction $5134-42$

King RS, Anderson SH and Killian GJ (1994) Effect of bovine oviductal estrus-associated protein on the ability of sperm to capacitate and fertilize oocytes Journal of Andrology 15 468-478

Komiya H, Onuma T, Hiroi M and Araki Y (1996) In situ localization of messenger ribonucleic acid for an oviduct-specific glycoprotein during various hormonal conditions in the golden hamster Biology of Reproduction 55 1107-1118

*Kouba AJ, Abeydeera LR, Alvarez IM, Day BN and Buhi WC (2000) Effects of the porcine oviduct-specific glycoprotein on fertilization, polyspermy, and embryonic development in vitro. Biology of Reproduction 63 242-250

Lagow E, DeSouza MM and Carson DD (1999) Mammalian reproductive tract mucins Human Reproduction Update 4 280-292

Leese HJ (1988) The formation and function of oviduct fluid Journal of Reproduction and Fertility 82 843-856

McCauley TC, Buhi WC, Didion BA and Day BN (2001) Exposure of oocytes to porcine oviduct-specific glycoprotein reduces the incidence of polyspermic penetration in vitro. Sixth International Conference on Pig Reproduction 47

Malette B and Bleau G (1993) Biochemical characterization of hamster oviductin as a sulfated zona pellucida-binding glycoprotein Biochemical Journal 295 437-445

*Martus NS, Verhage HG, Mavrogianis PA and Thibodeaux JK (1998) Enhancement of bovine oocyte fertilization in vitro with a bovine oviductal specific glycoprotein Journal of Reproduction and Fertility $\mathbf{1 1 3}$ 323-329

Merlen Y and Bleau G (2000) Organization of a gene coding for an oviductspecific glycoprotein (oviductin) in the hamster Molecular Reproduction and Development $\mathbf{5 7} 238-246$

Murray MK and DeSouza MM (1995) Messenger RNA encoding an estrogen-dependent oviduct secretory protein in the sheep is localized in the apical tips and basal compartments of fimbria and ampulla epithelial cells implying translation at unique cytoplasmic foci Molecular Reproduction and Development 42 268-283 
O'Day-Bowman MB, Mavrogianis PA, Reuter LM, Johnson DE, Fazleabas AT and Verhage HG (1996) Association of oviduct-specific glycoproteins with human and baboon (Papio anubis) ovarian oocytes and enhancement of human sperm binding to human hemizonae following in vitro incubation Biology of Reproduction 54 60-69

Oliphant G, Reynolds AB, Smith PF, Ross PR and Marta JS (1984) Immunocytochemical localization and determination of hormoneinduced synthesis of the sulfated oviductal glycoproteins Biology of Reproduction 31 165-174

*Paquette Y, Merlen Y, Malette B and Bleau G (1995) Allelic polymorphism in the hamster oviductin gene is due to a variable number of mucin-like tandem repeats Molecular Reproduction and Development 42 388-396

Robitaille G, St-Jacques S, Potier M and Bleau G (1988) Characterization of an oviductal glycoprotein associated with the ovulated hamster oocyte Biology of Reproduction 38 687-694

Satoh T, Abe H, Sendai Y, Iwata H and Hoshi H (1995) Biochemical characterization of a bovine oviduct-specific sialo-glycoprotein that sustains sperm viability in vitro. Biochimica et Biophysica Acta 1266 117-123

Sendai Y, Abe H, Kikuchi M, Satoh T and Hoshi H (1994) Purification and molecular cloning of bovine oviduct-specific glycoprotein Biology of Reproduction 50 927-934

Sendai Y, Komiya H, Suzuki K, Onuma T, Kikuchi M, Hoshi H and Araki Y (1995) Molecular cloning and characterization of a mouse oviductspecific glycoprotein Biology of Reproduction 53 285-294

Shemesh M (2001) Actions of gonadotrophins on the uterus Reproduction $121835-842$

Sun T, Lei ZM and Rao CV (1997) A novel regulation of the oviductal glycoprotein gene expression by luteinizing hormone in bovine tubal epithelial cells Molecular and Cellular Endocrinology 131 97-108

Sutton R, Nancarrow CD, Wallace ALC and Rigby NW (1984) Identification of an oestrus-associated glycoprotein in oviductal fluid of the sheep Journal of Reproduction and Fertility 72 415-422

Suzuki K, Sendai Y, Onuma T, Hoshi H, Hiroi M and Araki Y (1995) Molecular characterization of a hamster oviduct-specific glycoprotein Biology of Reproduction 53 345-354

*Takahashi K, Sendai Y, Matsuda Y, Hoshi H, Hiroi M and Araki Y (2000)
Mouse oviduct-specific glycoprotein gene: genomic organization and structure of the 5'-flanking regulatory region Biology of Reproduction $\mathbf{6 2}$ 217-226

Verhage HG, Fazleabas AT and Donnelly K (1988) The in vitro synthesis and release of proteins by the human oviduct Endocrinology 122 1639-1645

Verhage HG, Boice ML, Mavrogianis P, Donnelly $K$ and Fazleabas AT (1989) Immunological characterization and immunocytochemical localization of oviduct-specific glycoproteins in the baboon (Papio anubis) Endocrinology 124 2464-2472

Verhage HG, Mavrogianis PA, Boice ML, Li W and Fazleabas AT (1990) Oviductal epithelium of the baboon: hormonal control and the immunogold localization of oviduct-specific glycoproteins American Journal of Anatomy 187 81-90

Verhage HG, Mavrogianis PA, Boomsma RA, Schmidt A, Brenner RM, Slayden OV and Jaffe RC (1997a) Immunologic and molecular characterization of an estrogen-dependent glycoprotein in the rhesus (Macaca mulatta) oviduct Biology of Reproduction 57 525-531

Verhage HG, Fazleabas AT, Mavrogianis PA, O'Day-Bowman MB Donnelly KM, Arias EB and Jaffe RC (1997b) The baboon oviduct: characteristics of an oestradiol-dependent oviduct-specific glycoprotein Human Reproduction Update 3 541-552

Vieira EG, Chapman DA and Killian GJ (1999) Evidence for multiple forms of estrus-associated protein in bovine oviductal fluid associated with oviduct region and stage of the estrous cycle Biology of Reproduction $\mathbf{6 0}$ (Supplement 1) 202

Wallenhaupt K, Alm H, Tomek W and Brussow K-P (1996) Studies on the influence of a specific $97-k d$ porcine oviductal secretory protein on the de novo protein synthesis of preimplantation embryos Archives of Animal Breeding 3974

Watanabe T, Kobori K, Miyashita K, Fujii T, Sakai H, Uchida M and Tanaka H (1993) Identification of glutamic acid 204 and aspartic acid 200 in chitinase A1 of Bacillus circulans WL-12 as essential residues for chitinase activity Journal of Biological Chemistry 26818 567-18 572

Wegner CC and Killian GJ (1991) In vitro and in vivo association of an oviduct estrus-associated protein with bovine zona pellucida Molecular Reproduction and Development 29 77-84 